quite firmly the earth in the cylinders. They may then be fumigated with oarbon bisulphide to kill any creatures which might attack the puparia of the Tachinids, and yhen they have been afterward thoroughly aired, the Tachinid lane as they emerge from their hosts, may be placed in them and allowed to pupate as they wish. This may be facilitated by placing material suspected to contain thachinids upon a piece of mosquito netting stretched above the earth in a cylinder in the manner already described earlier in this paper. The cylinder may then be sunk in the ground in some cool, moist place, examined from time to time in the summer to secure the summer-issuing species and again thext spring for the hibernating forms. The essential thing is the the earth in which the maggots have pupated be kept moist during the winter in order to successfully rear the hibernating species. With the forms which hibernate within the body of the over-wintering host, the methods which suffice to carry the latter to maturity will suffice for the parasites. It is only necessary to recognize that such hibernating hosts may contain Tachinid parasites whose habits and identity it is well to determine.

\title{
FOOD OF THE BOBWHITE
}

By Margaré Morse NICE, Clark University, Worcester, Mass.

\section{INTRODUCTORY}

To become effective, conservation of our valuable bird life must be based on definite knowledge of the facts. To gather the facts requires patient study, and the present paper gives the results of more than two years research and presents the most complete and convincing statement that we have of the foo of any bird. As these results become generally known, by sheer weight of values involved, they will put the bobwhite, properly appreciated and protected, in every farm and garden in the land.

This paper forms a part of a more complete monograph on the Biology of the Bobwhite, which aims to discuss the species in all its complex relations to the life of the continent. Two general points, however, may be anticipated.

First, former researches have demonstrated that the bobwhite feeds almost entirely on weed seeds and insects, and that it does, no appreciable damage to agriculture. Some may ask for a montated analysis of the insect food and may question whether for may not take valuable insects to such an extent as to ded 
the account in its favor. To do our work we are at liberty to choose among the many forces of living nature those most effective, most easily controlled and most agreeable. On all these counts, for destruction of weed seeds and insects, the bobwhite leaves nothing to be desired. A bird that takes so many injurious insects is welcome to the beneficial ones as well. Apparently, if we could have enough bobwhites, they would leave nothing for the beneficial insects to do.

Second, the tendency at present, over a large portion of its natural range is strongly toward extinction of the bobwhite. To reverse this tendency will require careful study of the problems involved and vigorous and concerted effort. It is generally recogaized that extermination of natural enemies is the beginning of game protection. For the bobwhite, cats go farther toward accounting for scarcity and extinction of the birds than sportsmen and all other natural enemies combined. Crows, rats, skunks, weasels, minks and several of the hawks and owls, especially the three first, are enemies which must be controlled, if the species is to increase anywhere, or if the birds are even to hold their own; and no one should attempt to introduce stock for the purpose of colonization until the ground has been thoroughly gone over and all vermin exterminated. Although protected by cages, cats have repeatedly broken up nests by frightening off the brooding birds at night, and in one instance a cat disturbed a pair with a brood of fifteen chicks and all but three were dead next morning, chilled in the wet grass. One such occurrence shows that the damage a cat may do is only limited by the number of birds she is able to find. She might as easily have scared up a dozen broods in a night as one, and still, with cats ranging at will everywhere, we pretend to wonder why the bobwhite is so scarce.

The paper is, further, an illustration of the possibilites of investigat. ing the food of a species by the feeding test method. The striking feature is the quantitative results, the day's works, but qualitatively also the fact that so many species could be added to the dietary of a bird already so carefully studied by the method of crop examination suggests that the feeding test method may with profit be applied to many species of birds. The method is sure to yield in the near future much more complete results on the side of insects destroyed. In fact, many species of insects were eaten, as they were swept up in the nets, that were not definitely identified.

The present paper is printed in the hope of educating the public and of furnishing support for the strong movement already afoot for the more adequate protection of the bobwhite. It would seem that all who read it must agree with the author's conclusion : 
"If we were wise enough as a people to protect and increase our weed-destroying and insectivorous birds, they should largely control these enemies of our crops. Bobwhites, if we only had enough of them, ought to save us more than half of our $\$ 17,000,000$ weed damage and of our billion dollar insect tax."

\section{F. HOMGE.}

The experiments have been carried on since September, 1907, in Worcester, Mass., with birds that had been under domestication three and four generations. The original stock came from Kansas, Alabama and South Carolina. Eighteen birds of the third generation were used, chiefly for weed tests; some were hatched under hens, some in an incubator; all were raised in brooders. A special study was made in 1908 of one bobwhite of the next generation.

\section{WEED SEEDS}

Dr. Sylvester Judd of the Bureau of Biological Survey has made a careful study of the food of the Bobwhite by analysis of stomach contents. In his bulletin "The Bobwhite and Other Quails of the United States in their Economic Relations" he says:

"The bobwhite is preëminently a seed eater, 52.83 per cent of its food for the year consisting of seeds. The bulk of these are the seeds of plants belonging to the general category of weeds. The food of no other bird with which the writer is acquainted is so varied." (6)

"The laboratory work to determine the different kinds of food and their proportions has included examination of crops and gizzards from 918 birds. This material was collected from 21 States, Canada, the District of Columbia, and Mexico, but chiefly from New York, Maryland, Virginia, Florida, Illinois, South Dakota, Nebraska, Kansas, and Texas. Stomachs were obtained each month of the year, but unfortunately few were collected in the breeding season. Laboratory work included also feeding experiments with three pairs of captive bobwhites obtained from Kansas."

In my experiments the weeds or merely the seeds were put into the cages, to see whether the birds would eat them. By this means 61 weeds were added, making 129 . Dr. Judd's contributions to the following list are starred.

\section{WEED SEEDS EATEN BY BOBWHITE}

*Barbed panicum................Panicum barbatum.

*Barnyard gress, barn grass, cocks-

foot, water grass................ Panicum crusgalli.

*Bastard pennyroyal...............Trichostema dichotomum. 
*Beggarticks, bur marigold, pitchforlis, stick seed................Bidens sp.

*Bindweed, bear bind, English bindwoed, morning glory...............Convolvulus arvensis.

*Black bindweed...................Polygonum convolvulus.

Black mustard, brown mustard, grocer's mustard.................... Brassica nigra.

Blue vervain, simpler's joy...........Verbena hastata.

Blue weed.....................Echium vnilgare.

Boneset, ague weed, fever weed, thoroughwort ................. Eupatorium perfoliatum.

Bouncing Bet, hedge pink, saapwort....Saponaria officinalis.

Bracted plaintain, western plaintain...Plantago aristata.

Bull thistle, bird thistle, boar thistle, pasture thistle................. Cardurs lanceolatus.

Burdock, beggar's buttons, gobo, great dock ...................... Artium lappa.

Butter and eggs, toadflax, devil's flax, snapdragon ................. Linaria linaria.

"Button weed, compass weed, poor weed.. Diodia teres.

Canada thistle, creeping thistle, cursed thistle ................. Carduns arvensis.

*Carpet weed, Indian chickweed........Mollugo verticillata.

*Charlock, wild mustard, yellow mustard ....................... Raphanus raphanistrum.

*Chickweed, common chickweed........Alsine media.

Chicory, savory................ Chicoritum intybus.

*Climbing false buckwheat, bindweed.... Polygonum scandens.

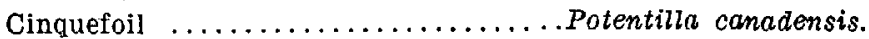

Common darnel ................. Lolium temulentum.

*Corn cockle, bastard nigella, cockle, rose campion...................... Arostemma githago.

*Corn gromwell, field gromwell, red root, wheat thief................ Lithospermum arvense.

* Crab grass, finger grass, Polish millet..Panicum sanguinale.

*Creeping bush clover...............Lespedeza repens.

*Croton ....................... Croton sp.

*Crownbeard .................... Verbesina sp.

*Curled dock, sour dock, yellow dock... Rumex crispus.

Dandelion .................... Taraxicum taraxicum.

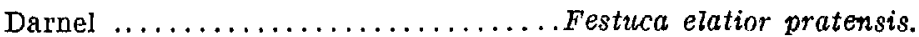

Dodder ................................... gronovii.

Evening primrose ...............Enothera biennis.

*Everlasting ..................... Antennaric sp.

False flax, gold of plensure, Siberian oilseed, wild flax............... Camelina sativa.

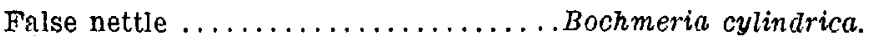

Fireweed $\ldots \ldots \ldots \ldots \ldots \ldots \ldots \ldots$. Erechtites hieracifolia.

*Flowering spurge, showy spurge.....Euphorbia corollata.

Fringed black bindweed............ Polygonum cilinode. 
-Giant ragweed, hogweed, horseweed,

tall ragweed .................... Ambrosia trifida.

*Green foxtail, green pigeon grass, bottle grass...................Chcetochlia viridis.

*Gromwell ...................... Lithospermum officinale.

*Hairy bush clover................Lespedeza hirta.

Fledge mustard ................ Sisymbrium officinale.

*Hoary puccoon ................... Lithospermum canescens.

*Hoary vervain ................. Verbena stricta.

Horse nettle, bull nettle, radicle, sand briar ...................... Solanum carolinense.

Horseweed, butterweed, colt's tail, fleabane .................... Erigeron canadense.

Ironweed $\ldots \ldots \ldots \ldots \ldots \ldots \ldots$. . . . . . . . . . .

*Japan Clover .....................espedeza striata.

*Jewel weed, touch-me-not...........Impatiens sp.

Joe-Pye weed, trumpet weed...........Eupatorium purpureum.

*Knotweed, doorweed, goose grass......Polygonum aviculare.

*Lamb's-quarters, goosefoot, pigweed..Chenopodium album.

*Lupine .......................Lupinus sp.

* Marsh elder, false ragweed, false sunflower, high-water shrub.......... Iva xanthifolia.

Mayweed, dog fennel, stinking chamo-

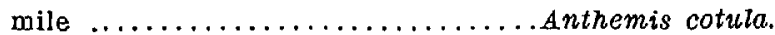

Meadowsweet..$\ldots \ldots \ldots \ldots \ldots \ldots$. Spircea salcifolia.

- Milk purslane, spotted spurge........ Euphorbia maculata.

Milkweed, silkweed, wild cotton........Asclepias syriaca.

*Moraing glory ................. Ipomoea sp.

Motherwort ................... Leonurus cardiaca.

Mouse-ear chickwęed............... Cerastium vulgatum.

Mullein, Aaron's rod, flannel plant, velvet dock.................... Verbascum thapsus.

- Nightshade, deadly or black-berried nightshade ................. Solanum nigrum.

Nonesuch, black medick, medicago.....Medicago lupulina.

*Nut grass, coco, coco sedge, nutsedge... Cyperus rotundus.

*Old witch grass ................Panicum capillare.

* Orange hawkweed, devil's paint brush, golden hawkweed ...........Hieracium aurantiacum.

Ox-eye daisy, bull's-eye, white daisy,

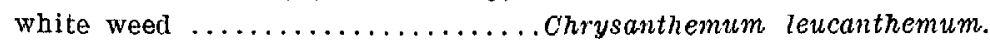

*Partridge pea ....................Chomocrista fascicularis.

*Pennsylvania persicaria..............Persicaria pennsylvanica.

Pepper grass ................... Lepidium virginicum.

*Persicaria, pale..................Persicaria lopathifolia.

*Pigeon grass, pussy grass, summer or yellow foxtail .................. Chatochloa glauca.

"Pigweed, redroot, rough amaranth......Amaranthus retroflexus.

Plantain, white man's loot............ Plantago major.

*Poison ivy, poison vine............ Rhus radicans.

Pokeweed, garget, pigeon berry, slioke..Phytolacca decandra. 
Prickly lettuce, strong-scented lettuce, ,Lactuca virosa.

Purslane, garden purslane, pursley, pusley ........................ Portulaca oleracea.

Rabbit's-foot clover, stone clover......Trifolinm arvense.

*Ragweed, bitterweed, hogweed, Roman wormwood, richweed .............. Ambrosia artemisicefolia.

*Ribgrass, black plantain, buck holn, deer tongue....................Plantago lanceolata.

Rough avens...................Gentm virginianum.

Found-headed bush clover............ Lespedeza capitato.

Round-leafed mallow, cheeses, mal-

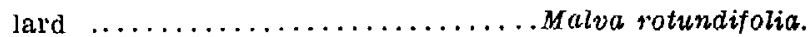

Russian pigweed.................. Axyris amarantoides.

* Sedge $\ldots \ldots \ldots \ldots \ldots \ldots \ldots \ldots \ldots$ Carex sp.

* Sensitive pea...................Chomocrista nictitans.

* Sheathed rush grass..............sporobolus vaginceflorus.

Shepherd's purse, mother's heart, pickpurse, toothwort............Birsa bursa-pastoris.

* Sida ....................... Sida spinosa.

*Skunk cabbage................. Spathyema fatida.

Skunk tail grass................... Hordeum jubatum.

* Slender finger grass................ Syntherisma filiformis.

*Slender paspalum..................Paspahum setaceum.

*Slender spike grass................ Uniola laxa.

* Smartweed ................... Polygonum hydropiper.

* Sorrel: field, horse, red or sheep sorrel; sour weed ...............Rumex acetosella.

*Spreading panicum...............Panicum proliferum. Spurry ....................... Spergota atvensis. Steeplebush .................. Spirca tomentosa, Sticktight, beggar's lice.............Lappula virginiana. Stinkweed, penny-cress, French weed..Thlaspi arvense. St. John's wort.................. Hyperium perforatum. * Sunflower ...................... Helianthus annuus.

Sweet grass....................Hierochloë vorealis.

*Switch grass, tall smooth panicum.....Panicum virgatum.

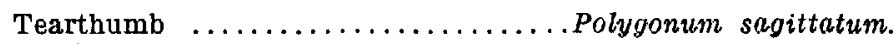

*Texas croton........................ Croton texensis.

*Three-seeded mercury, copper-leaf.....Acalypha glacilens.

*Tick-trefoil .....................Meibomia grandiflora.

"Tick-trefoil .....................Meibomia nudifora.

*Trefoil ........................ Lotus sp.

*Tussock sedge....................... sarex stricta.

-Vetch ........................

Water hoarhound.................Lycopus americanus.

Water smartweed.................Polygonum acre.

White vervain, nettleleaved vervain...Verbena stricta.

Wild carrot, bird's nest, lace weed;

Queen Anne's lace................ Daucus carota.

Wike oats.......................

Wild rice ...................... Zizania aquatica. 
Witch grass.........................

Yarrow, milfoil.....................chillea millefolium.

Yellow daisy, brown-eyed Susan, cone

flower .....................Rudbeckia hirta.

*Yellow sorrel................... Oxalis stricta.

\section{Feeding Habits}

Another experiment was an attempt to bring up a bobwhite in an entirely natural way so far as weeds were concerned. When he was a month old, he was taken into the garden or fields every few days, and watched to see what he would eat. He was given no weed seeds until after he had found and eaten them out-of-doors. He would experiment on many things that were not eatable, and if he had been out for half a day instead of less than an hour at a time, and if he had been taken to more places to find weeds, undoubtedly he would have eaten a greater variety.

The following seeds were his special favorites:

Barnyard grass.

Chickweed.

Pigeon grass.

Ragweed.

Yellow sorrel.

He was also fond of

Cinquefoil.

Lamb's quarters.

Peppergrass.

Pigweed.

Plantain.

Rabbit's foot clover.

Red sorrel.

In regard to the number of seeds of certain weeds that a bobwhite will eat at a meal, Dr. Judd has several records of the amounts found in single crops. In a few cases $I$ watched a bird eat all that he wanted counting while he ate.

\begin{tabular}{|c|c|c|c|c|}
\hline Chickweed & 2,025 & 2,250 & Russian pigweed & 350 \\
\hline *Crab grass & 1,000 & & *Smartweed & 300 \\
\hline *Lamb's quarters & & 10,000 & *Sorrel & 550 \\
\hline *Pigeon grass & & 5,000 & Stinkweed & 10 \\
\hline *Pigweed & & 400 & Sweet grass & 200 \\
\hline *Ragweed & & 1,000 & & \\
\hline
\end{tabular}

In order to find out how many seeds of one kind a bobwhite would eat in a day the following tests were made. A weighed amount of 
clean weed seeds was put into a box, which was set inside a larger box, so that any seed scratched out would be caught and all that the birds did not eat, weighed. One gram of each kind of seed was counted. Two birds were used in each feeding test; they had nothing but green food to eat besides the weed seeds.

NUMBER OF SEEDS EATEN BY A BOBWHITE IS A DAY

$\begin{array}{lrlr}\text { Barnyard grass } & 2,500 & \text { Milkweed } & 770 \\ \text { Beggar ticks } & 1,400 & \text { Peppergrass } & 2,400 \\ \text { Black mustard } & 2,500 & \text { Pigweed } & 12,000 \\ \text { Burdock } & 600 & \text { Plantain } & 12,500 \\ \text { Crab grass } & 2,000 & \text { Rabbit's foot clover } & 30,000 \\ \text { Curled dock } & 4,175 & \text { Round headed bush clover } & 1,800 \\ \text { Dodder } & 1,560 & \text { Smartweed } & 2,250 \\ \text { Evening primrose } & 10,000 & \text { White vervain } & 18,750 \\ \text { Lamb's quarters } & 15,000 & & \text { Water smartweed } \\ & & & 2,000\end{array}$

To quote again from Dr. Judd:

"A careful computation of the total amount of weed seed the bob. white is capable of destroying is surprising in the magnitude of its result. In the State of Virginia it is safe to assume that from September 1 to April 30, the season when the largest proportion of weed seed is consumed by birds, there are four bobwhites to the square mile, or 169;800 in the entire State. The crop of each of these birds will hold half an ounce of seed, and as at each of the taily meals weed seed constitutes at least half the contents of the crop, or a quarter of an ounce, a half ounce daily is certainly consumed by each bird. On this very conservative basis the total consumption of weed seed by bobwhites from September 1 to April 30 in Virginia amounts to 573 tons."

The following tests were made in order to ascertain how much bobwhites eat each day.

\begin{tabular}{|c|c|c|c|c|c|c|c|c|c|c|c|}
\hline $\begin{array}{l}\text { Four } \\
\text { Two }\end{array}$ & $\begin{array}{l}\text { birds ate } \\
\text { birds ate }\end{array}$ & $\begin{array}{l}60 \\
30\end{array}$ & $\underset{\text { grams }}{\sin }$ & & $\begin{array}{l}\text { weed } \\
\text { " }\end{array}$ & $\begin{array}{l}\text { seeds } \\
\text { “ }\end{array}$ & & le day. & $\begin{array}{l}\text { Indoors, } \\
\text { ": }\end{array}$ & & $\begin{array}{l}\text { November } \\
\text { “a }\end{array}$ \\
\hline One & bird ate & 16.5 & grams & of & weed & seeds & and & grain. & Indbors, & in & December. \\
\hline One & bird ate & 17 & $"$ & $"$ & " & $"$ & " & “ & “ & “ & October. \\
\hline One & bird ate & 14 & $"$ & “ & $" ،$ & " & $“$ & " & “ & " & " \\
\hline One & bird ate & 20 & $"$ & " & “" & $"$ & “ & " & is & $"$ & $"$ \\
\hline One & bird ate & 15 & "s & “ & $"$ & " & “ & " & $\because$ & “ & “ \\
\hline One & bird ate & 14 & 't & “ & " & " & “* & $"$ & $"$ & “ & “ \\
\hline One & bird ate & 21 & "6 & “ & $"$ & $"$ & * & " & $" 4$ & $"$ & “ \\
\hline One & bird ate & 17 & " & $"$ & “ & " & “ & “ & $"$ & $“$ & “ \\
\hline One & bird ate & 14 & " & “ & " & " & " & “ & " & $"$ & " \\
\hline One & bird ate & 12 & " & $"$ & “ & " & $"$ & "، & " & $“$ & $"$ \\
\hline One & bircl ate & 17 & “ & “ & “. & “ & “ & " & “ & “ & " \\
\hline
\end{tabular}


One bird ate 17 grams of weed seeds and grain. Indoors, in October.

One bird ate 12

One bird ate 13

" “ “

Twelve birds ate 180 grams of grain in one day. Outdoors, in February.

Twelve birds ate 180 grams of weed seeds in one day. Outdoors, in February.

Twelve birds ate 180 grams of grain in one day. Outdoors, in February.

The average of these tests is 15 grams, a little more than half an ounce.

\section{INSECTS}

"The bobwhite eats insects in every month of the year. Moreover the large proportion of injurious insects habitually eaten renders the services of this bird more valuable than those of many birds whose percentage of insect food, though greater, includes a smaller proportion of injurious species. Conspicuous among the pests destroyed are the Colorado potato beetle, twelve-spotted cucumber beetle, bean leaf-beetle, squash ladybird, wireworms and their beetles, and May beetles. Its food also includes such weevils as corm billbugs, imbricated snout beetle, clover leaf weevil, cotton boll weevil; also the striped garden caterpillar, army worm, cotton bollworm, and various species of cutworms; also the corn-louse ants, red-legged grasshopper, Rocky Mountain locust, and chinch bug." (9)

The most important insects added by my experiments are the squash bug, plant lice, the cabbage butterfly, cankerworms, codling moth, the Hessian fly, the mosquito, stable fly, and the typhoid fly.

Thysanura

LIST OF INSECTS EATEN BY THE BOBWHITE.

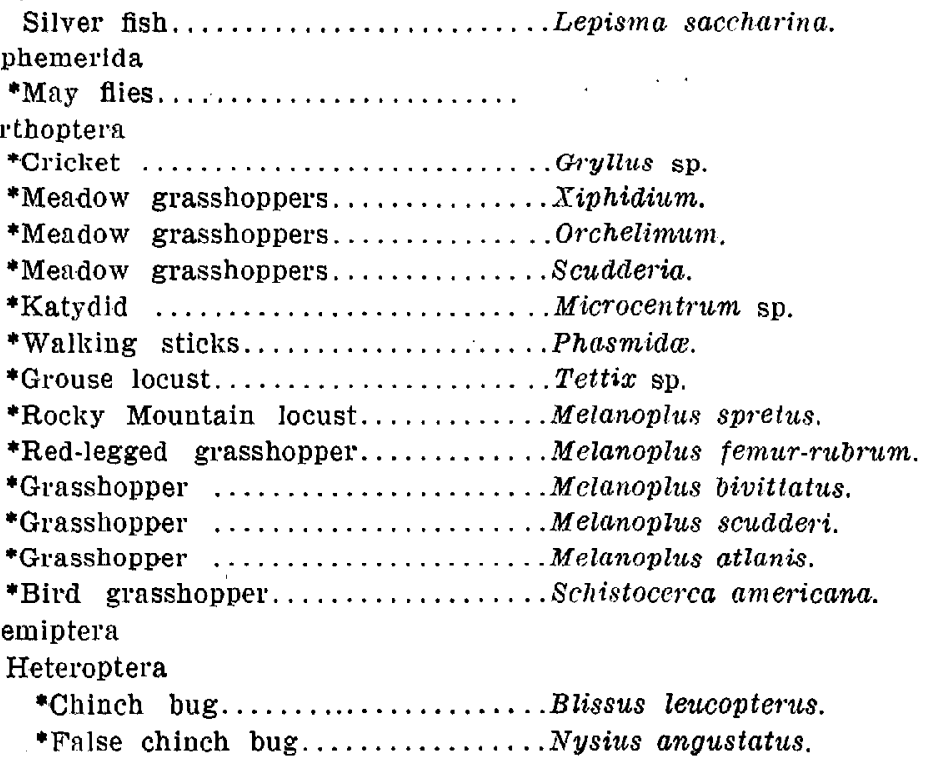


*Three-spotted soldier bug.......Euschistus tristigmus.

* Stink bug.................Euschistus variolarius.

*Stink bug..................Euschistus sp.

* Bug .................... Podisus sp.

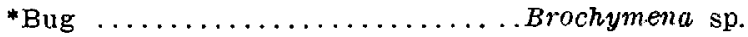

${ }^{*}$ Bug $\ldots \ldots \ldots \ldots \ldots \ldots \ldots \ldots$ Nezara hilaris.

*Bug . .........................

*Bug ...................... Hymenarcis nervosa.

*Bug ........................menarcis cqualis.

*Bug .................... Thyanta custator.

*Bug ..................... gabalus pugnax.

*Bug ..................... Trichopepla semivittata.

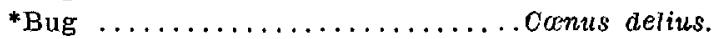

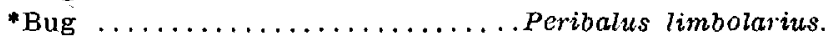

*Tarnished plant bug............. Lygus pratensis.

${ }^{*}$ Bug . . . . . . . . . . . . . . Corimelcena sp.

${ }^{*}$ Bug $\ldots \ldots \ldots \ldots \ldots \ldots \ldots \ldots$ Apiomerus crassipes.

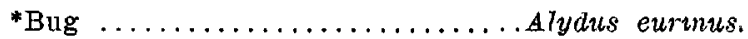

${ }^{*}$ Bug $\ldots \ldots \ldots \ldots \ldots \ldots \ldots \ldots \ldots$ Corizus sp.

*Bug ..................... Euthocta galeator.

*Shield-backed bugs.............Scutellerida.

Squash bug.................. Anasa tristis.

Homoptera

*Leaf hopper.................. Oncometopia lateralis.

*Leaf hopper.................. Oncometopia sp.

*Leaf hopper................. Deltocephalus sp.

*Leaf hopper.................. Diedrocephala sp.

Plant lice..................... Aphidide.

Tree hoppers.................Membracide.

Lepidoptera

*Army worm................. Heliophila unipuncta.

*Cutworm ..................... Agrotis sp.

*Cutworm ...................... Feltia annexa.

*Noctuid moth.................. Noctuida.

*Cotton worm..................... Alabama argillacea.

*Cotton bollworm................Heliothis obsoleta.

* Striped garden caterpillar...........Mamestra legitima.

*Yellow bear caterpillar............ Diacrisia virginica.

*Pyralid ..................... Tholeria reversalis.

*Purslane sphiux..............Deilephila gallii.

* Southern tobacco worm............Phlegethontius sexta.

*Caterpillar ..................Junonia cœnia.

*Pupa ..................... Vanessa sp.

Cabbage butterfly ............... Pieris rapa.

Canker worms.................

Tent caterpillar............... Clisiocampa americana.

Bee moth .................Galleria melonella.

Codling moth.................. Carpocapsa pomonella.

Clothes moth................. Tinea pellionella.

\section{Diptera}

*Crane fly Tipulider. 
*Green fly

Lucilia cosar.

* Robber fly

Asilida.

Mosquito

Culex.

Mosquito

Anopheles.

Hessian fly......................

Typhoid fly...................Musca domestica.

Stable fly.................... Stomoxys calcitrans.

Coleoptera

Carabidæ

"Ground beetle.................Scarites subtervaneus.

*Ground beetle.................Amara sp.

*Ground beetle.................Casnomia pennsylvanica.

*Ground beetle.................Platynus extensicollis.

*Ground beetle.................. Agonoderus pallipes.

*Ground beetle.................Harpalus pennsylvanicus.

*Ground beetle................Harpalus calignosus.

*Ground beetle.................Anisodactylus rusticus.

*Ground beetle................. Anisodactylus baltimorensis.

Chrysomelidæ

*Leaf beetle.................. Cryptocephalus venustus.

* Leaf beetle..................... Colapsis brunnea.

- Leaf beetle.................... Nodonota tristis.

- Leaf beetle....................Chrysomela pulchra.

*Leaf beetle...................Chrysomela suturalis.

*Leaf betle.................... dionychis fimbriata.

*Leaf beetle.................... Disonycha 5-vittata.

*Leaf beetle.................. Disunycha xanthomelcena.

*Leaf beetle.....................Disonycha crenicollis.

*Leaf beetle...................Psylliodes punctulata.

*Leaf beetle...................Microrhopala vittata.

*Three-lined potato beetle......... Lema trilineata.

* Colorado potato beetle............ Leptinotarsa decemlineata.

-Bean leaf beetle.................Cerotoma trifurcata.

*Striped cucumber beetle...........Diabrotica vittata.

*Twelve-spotted cucumber beetle.... Diabrotica 12-punctata.

*Locust leaf-mining beetle......... Odontota dorsalis.

*Golden tortoise beetle............Coptocycla bicolor.

Elm-leaf beetle.................Galemicella Iuteola.

Scarabæidæ

*May beetle ..................Lachnosterna tristis.

*Dung beetle................. Onthophagus pennsylvanicus.

*Dung beetle................ Aphodius inquinatus.

*Leaf-chafer .................. Diplotaxis sp.

* May beetles....................Serica sp.

*May beetles...................Anomala sp.

*May beetles...................Aphonus sp.

Rhynchophora

*Imbricated snout beetle.........Epiccemus imbricatus.

*Iruller's rose beetle.............Aramigus fulleri.

* Clover weevil................... Sitones hispidulus.

*Clover-leaf weevil...............Phytonomus punctatus.

* Mexican cotton boll weevil........Anthonomus grandis. 
*Billbug ...................Sphenophorus parvnlus.

*Corn billbug.................. Sphenophorus zece.

*Weevil ....................Thecesternus humeralis.

*Weevil ................... Tanymecus confertus.

*Weevil .....................Chalcodermus collaris.

*Weevil .................. Centrinus. sp.

Elateridæ

${ }^{*}$ Click beetle................. Drasterius elegans.

*Click beetle....................Agriotes sp.

${ }^{*}$ Click beetle..................... Melanotus oommunis.

*Click beetle.................. Corymbites sp.

\section{Coceinellidæ}

*Lady beetle .................Hippodamia parenthesis.

*Squasl ladybird............... Epilachna borealis.

*Lady beetle ................. Coccinella sanguinea.

"Lady beetle ..................Adalia bipunctata.

\section{Histeridæ}

*Histerid beetles

Tenebrionidæ

*Darkling beetle..............Blaptinus.

Menlworms ................. Tenebrio sp.

Staphylinidæ

*Rove beetles.

Lampyridæ

*Soldier beetle................Chauliognathus pennsylvanicus.

Cerambycidæ

*Longicorn beetle...............Tetraopes tetraophthalmis.

Dermestidæ

Carpet beetle................Anthrenus scrophularia.

Hymenoptera

*Ants ....................... Lasius sp.

*Ants ........................ Tetramorium ccespitum.

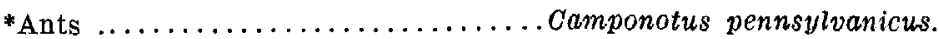

*Gall flies..................... Cynipide.

*Parasitic wasps................Tiphia inornata.

*Parasitic wasp..................Proctotrypes rufipes.

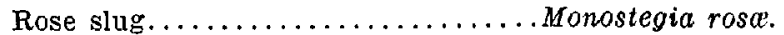

Currant worm.....................

Other animal food

*Spiders

* Harvest spiders................Phalangida.

*Thousand leg..................Julus sp.

Sow bug..................... oniscide.

*Snail ...................... Pupa armifera.

*Pond snail.................... Succinea avara.

*Crayfish .................... Cambarus.

*Toad

The following are a few records of the numbers of insects eaten by bobwhites at single meals.

*Grasshoppers: from 20 to 39. 
* Chinch bugs: 100, in another case two tablespoonfuls in a cup.

Squash bugs : $6,11,12$.

Aphids: 2,326 eaten by a week old chick; this was more than one meal, but was not all his insect food for that day.

*Army worm: 12.

*Cutworm : 12.

Hessian fly flaxseeds: 20.

Mosquitoes: 144 - a week old bird; 568 - a nearly grown bird in three hours. In both these cases the supply gave out, while the birds were still eager for more.

* Potato beetles : 75, 101.

May beetle grubs : 7,8 .

*Cotton boll weevil : 47 .

Sow bugs: 6 .

Miscellaneous insects : 1,400 in half a day - a laying hen the $23 \mathrm{rd}$ of June.

Tests to ascertain how many insects of one kind a bobwhite might eat in an entire day gave the following results. The birds had plenty of weed seeds, grain and green food, except in two cases in which the fact is indicated.

5,000 aphids - chrysanthemum black fly. Adult bird in December. This is the only case in which the insects were not individually counted; one thousand were counted and the rest estimated.

1,350 flies. A laying hen in July. About one fifth were maggots, the rest adults.

59 adult potato beetles. A laying hen; test by Mazie Hodge.

1,286 rose slugs. A laying hen; test by Mazie Hodge.

37 grasshoppers and 2,400 seeds of pigeon grass eaten apiece by two six weeks old birds in October.

43 grasshoppers and 2,100 seeds of pigeon grass eaten apiece by two six weeks old birds in October.

20 grasshoppers and 3,000 seeds of pigeon grass eaten apiece by two six weeks old birds in October.

65 large crickets eaten apiece by two seven weeks old birds in October. They had no weed seeds or grain.

84 large and middle-sized grasshoppers eaten apiece by two seven weeks old birds in October. They had no weed seeds or grain.

700 insects -300 of them grasshoppers. A laying hen in July. Their weight was 24 grams.

1,532 insects - 1,000 of them grasshoppers. A laying hen in July. Their weight was 24.6 grams. 
Eight tests were made with an adult cock in October and November.

$$
\begin{array}{llll}
28 & \text { grasshoppers }=11 \mathrm{~g} . & 14 \mathrm{~g} . \text { of grain. } & \text { Total }-25 \mathrm{~g} . \\
33 \text { grasshoppers }=15 \mathrm{~g} . & 13 \mathrm{~g} . \text { of grain. } & \text { Total }-28 \mathrm{~g} . \\
48 \text { grasshoppers }=19 \mathrm{~g} . & 10 \mathrm{~g} . \text { of grain. } & \text { Total }-29 \mathrm{~g} . \\
22 \text { grasshoppers }=12 \mathrm{~g} . & 16 \mathrm{~g} . \text { of grain. } & \text { Total }-28 \mathrm{~g} . \\
25 \text { grasshoppers }=12 \mathrm{~g} . & 11 \mathrm{~g} . \text { of grain. } & \text { Total }-23 \mathrm{~g} . \\
23 \text { grasshoppers }=12 \mathrm{~g} . & \text { 8g. of grain. } & \text { Total }-20 \mathrm{~g} . \\
20 \text { grasshoppers }=9 \mathrm{~g} . & 12 \mathrm{~g} . \text { of grain. } & \text { Total }-21 \mathrm{~g} . \\
25 \text { grasshoppers }=11 \mathrm{~g} . & 10 \mathrm{~g} . \text { of grain. } & \text { Tota }-21 \mathrm{~g} .
\end{array}
$$

\begin{tabular}{|c|c|c|c|c|c|}
\hline \multicolumn{6}{|c|}{ At hatching weighed $6 \mathrm{~g}$. } \\
\hline 5 days & 8 & & & & \\
\hline 7 & $\ldots$ & & \multicolumn{3}{|c|}{ Ate 2326 aphids and 20 mealworms. } \\
\hline 8 & 10.5 & & \multicolumn{3}{|c|}{$\begin{array}{l}\text { A te } 2526 \text { apdias and } 20 \text { mealworms. } \\
\text { A te } 7 \text { grams of insects. }\end{array}$} \\
\hline $\mathbf{g}$ & $12.5 \mathrm{~g}$. & Increase 2.5 & & & \\
\hline 10 & 13. & .5 & & & \\
\hline 11 & 13.3 & $\mathbf{s}$ & \multicolumn{3}{|c|}{ Ate 380 insects. } \\
\hline 12 & 14. & .7 & & & \\
\hline 13 & 15. & 1. & \multicolumn{3}{|c|}{ Ate 514 insects. } \\
\hline 14 & 15.5 & .5 & \multicolumn{3}{|c|}{225 insects $=8 \mathrm{~g}$. A te 514 of his weight. } \\
\hline *15 & 15. & -.5 & 236 & 7 & 474 \\
\hline *16 & 14.8 & -.2 & 400 & 8 & 544 \\
\hline$* 17$ & 15.8 & 1. & 200 & 8 & $50 \%$ \\
\hline 18 & 16.5 & .7 & 851 & 8 & $48 \%$ \\
\hline 19 & 17.5 & 1. & 411 & 6.6 & $38 \%$ \\
\hline 20 & 20. & 2.5 & 354 & 10.1 & 504 \\
\hline 21 & 21.2 & 1.2 & 732 & 10 & $47 \%$ \\
\hline 22 & 22.5 & 1.3 & 287 & 10.4 & $46 \%$ \\
\hline 23 & 25.3 & 2.8 & 296 & 11 & 434 \\
\hline 24 & 26.2 & .9 & 185 & 8 & $80 \%$ \\
\hline 25 & 28.5 & 2.3 & 250 & 12 & $42 \%$ \\
\hline 26 & 30.5 & 2. & 393 & 9.3 & $30 x$ \\
\hline 27 & 32.6 & 2.1 & 529 & 10.2 & 314 \\
\hline 28 & 34.2 & 1.6 & 710 & 13.5 & $42 \%$ \\
\hline 35 & 43.5 & & & & \\
\hline 36 & 46.5 & 3. & & & \\
\hline 37 & 49.5 & 3. & 102 & 11. & $15 \mathrm{~g}$. of $\mathrm{grain}=26=52 \%$ \\
\hline 38 & 51. & 1.5 & 112 & 11. + & $12 \mathrm{~g}$. of grain $=23=45 \%$ \\
\hline 39 & 53. & 2. & 134 & $12.5+$ & $12 \mathrm{~g}$. of $\mathrm{grain}=21.5=464$ \\
\hline 44 & 67. & $\ldots$ & $\cdots$ & & \\
\hline 49 & 77. & $\ldots$ & $\cdots$, & & \\
\hline 54 & 91. & $\cdots$ & $\ldots$ & & \\
\hline 55 & 92. & $\cdots$ & $\ldots$ & & \\
\hline At 10 weeks & 123. & $\ldots$ & $\ldots$ & & \\
\hline 11 & 139. & 16. & $\cdots$ & & \\
\hline 12 & 153. & 14. & $\ldots$ & & \\
\hline 13 & 156. & 3. & $\ldots$ & & \\
\hline 14 & 164. & 8. & $\ldots$ & & \\
\hline 15 & 166. & 2. & $\cdots$ & & \\
\hline
\end{tabular}

The average is:

28 grasshoppers $=12.5 \mathrm{~g} . \quad 12 \mathrm{~g}$. of grain. Total $-24.5 \mathrm{~g}$.

STUDY OF THE GROWTH AND FEEDING OF ONE BOBWHITE

* The loss in weight is due to his having lice for three days.

At hatching he weighed $6 \mathrm{~g}$; in 9 days he had doubled in weight. In the next 2 weeks he had doubled again, and again at the end of another fortnight. It took him 3 more weeks then to weigh $96 \mathrm{~g}$.; in the 
last 8 weeks of his growth he gained $75 \mathrm{~g}$. For the first 12 weeks his average gain per day was $1.75 \mathrm{~g}$. The average daily gain each week was as follows: .5 g., .7 g., .8 g., 2.1 g., 1.6 g., 2.35 g., 2.4 g., 2.1 g., 2.1 g., 2.3 g., 2.3 g., 2 g. The next four weeks his growth was slow; the average daily gain each week was : $43 \mathrm{~g}$., $1.1 \mathrm{~g}$., $.3 \mathrm{~g} ., .7 \mathrm{~g}$.

At 4 months he weighed $170 \mathrm{~g}$. which is an average adult weight for him, although sometimes he has weighed from 12 to 19 grams more.

In every case he was weighed before he had had anything to eat. He always had all he would eat in the tests, but doubtless would have eaten somewhat more in a wild state, for he would have exercised more than he did when kept in confinement. I regret that I did not make feeding tests in the first two weeks of his life, but I do not think he ever ate more than two-thirds of his weight at the most. For the first two or three days the chicks eat little, as the unabsorbed yolk nourishes them. Charles W. Nash in "The Birds of Toronto" says:

"For the first two or three months of their lives young quail feed almost exclusively on insects, and each one will, while he is growing, consume nearly its own weight of them every day." (11)

Edward Harris states in "The Quail the Best Insect and Weed Exterminator Must be Better Protected" that:

"A young quail will consume its own weight in insects every day." (4)

My results do not agree with this. A bobwhite does not have to eat so much nor grow so fast as those birds that are helpless until they nearly reach adult size.

As a result of these tests I have made an estimate of the amount eaten by a bobwhite in a year.

First 2 weeks, $72 \mathrm{~g}=10,245$ insects. The 8 th day he ate $7 \mathrm{~g}$. Counting nothing for two days, we might assume he ate as follows: 4, 4, 4, $5,6,7,7,7,7,7,7,7,=72$. In four of these days he ate 3,415 insects. At the same rate in twelve he would have eaten 10,245.

$3 \mathrm{rd}$ and 4th weeks, $132 \mathrm{~g} .=5,334$ insects. Actual tests.

5th week, $88 \mathrm{~g} .=3,520$ insects. His average weight for this week was 38.8. A third of that multiplied by 7 should give an estimate of the amount eaten. With 40 insects to the gram, as was the case in the two weeks before, the result is 3,520 .

6 th week, $81 \mathrm{~g}=812$ insects $+91 \mathrm{~g}$. grain. In 3 days he ate $34.5 \mathrm{~g}$. $=348$ insects and $39 \mathrm{~g}$. of grain. The week is calculated at the same rate.

Totals: $373 \mathrm{~g}=10,911$ insects $+91 \mathrm{~g}$. grain.

In December, January, February, March, April and half of November the estimate is that found by 56 tests $-\mathbf{1 5} \mathrm{g}$. of weed seeds a day. 
ESTIMATE OF THE AMOUNT EATEN IN A YEAR

\begin{tabular}{|c|c|c|c|c|}
\hline & \multicolumn{2}{|c|}{ By an adult hen } & \multicolumn{2}{|c|}{ By an adult cock } \\
\hline & Insects & weeds & Insects & weeds. \\
\hline January ......... & ........ & $465 \mathrm{~g}$. & $\ldots \ldots$. & 4658. \\
\hline February...... & & 420 & & 420 \\
\hline March......... & . & 465 & . & 465 \\
\hline April........... & $90 \mathrm{~g}$. & 450 & $90 \mathrm{~g}$. & $\mathbf{4 5 0}$ \\
\hline May $\ldots \ldots \ldots \ldots \ldots \ldots \ldots \ldots$ & 372 & 372 & 372 & 372 \\
\hline June................... & 600 & 800 & 372 & 372 \\
\hline July...$\ldots \ldots \ldots \ldots \ldots \ldots$ & 620 & 310 & 372 & 872 \\
\hline August....................... & 620 & 310 & 372 & 872 \\
\hline September................ & 450 & 300 & 372 & 878 \\
\hline October.................. & 372 & $\mathbf{8 7 2}$ & 372 & 372 \\
\hline November.................. & 186 & 411 & 186 & 411 \\
\hline \multirow[t]{2}{*}{ December................... } & $\ldots \ldots \ldots \ldots \ldots \ldots \ldots$ & 465 & $\ldots \ldots \ldots+\ldots \ldots \ldots \ldots$ & 465 \\
\hline & $3310 \mathrm{~g}$. & $4640 \mathrm{~g}$. & $2508 \mathrm{~g}$. & $4908 \mathrm{~g}$. \\
\hline
\end{tabular}

For the cock in May, June, July, August, September, October and half of November, and for the hen in May, October and half of November the estimate is that found by 8 tests in October and November -12 g. of each. In' June, July and August when the hen is laying, $20 \mathrm{~g}$. of insects seems a safe average. In 2 tests in July a hen ate 24 g. and $24.6 \mathrm{~g}$. respectively.

For a young bird, if we substitute the figures for his first six weeks for six weeks of July and August in the adult cock's estimate, the result is $2,377 \mathrm{~g}$. of. insects and $4,495 \mathrm{~g}$. of weed seeds.

An average of 45 tests gave 22.5 insects to a gram. The highest is 60 in July, the lowest 2 in November. This average is too low for the smallest insects that the birds eat, such as plant lice, were not weighed; besides for the tests large insects were often caught in preference to smaller ones for convenience salie.

One gram of 23 different kinds of seeds were counted; the average was 1,096 .

$\begin{array}{lrlr}\text { Barnyard grass, } & 1,250 & \text { Evening's primrose, } & 2,000 \\ \text { Beggar's ticks, } & 200 & \text { Lamb's quarters, } & 1,000 \\ \text { Black bindweed, } & 300 & \text { Milkweed, } & 110 \\ \text { Black mustard, } & 500 & \text { Pepper grass, } & 3,000 \\ \text { Burdock, } & 100 & \text { Plgeon grass, } & 500 \\ \text { Crab grass, } & 500 & \text { Pigweed, } & 3,000 \\ \text { Curled dock, } & 835 & \text { Plantain, } & 2,500 \\ \text { Fringed black bindweed } & 250 & \text { Rabbit's foot clover, } & 2,000 \\ \text { Dodder, } & 780 & \text { Ragweed, } & 500\end{array}$




$\begin{array}{lrlr}\text { Round headed bush clover, } & 300 & \text { White vervain, } & 2,500 \\ \text { Smartweed, } & 900 & \text { Water smartweed, } & 200 \\ \text { Sorrel, } & 2,000 & \end{array}$

Thus a bobwhite cock might eat on an average in one year 56,430 insects and 5,379,168 weed seeds; a hen 74,475 insects and 5,063,520 weed seeds and a young bird 65,001 insects and 4,926,520 weed seeds.

Estimates of the Annual Loss Due to Weeds and Insects.

It is impossible to make calculations as to how much a bobwhite's eating of these thousands of insects and millions of weed seeds is worth to us in dollars and cents. I quote, however, a few estimates of the annual losses due to weeds and insects, so that we may get more of an idea of the importance of the problem.

"Since the total value of our principal field crops for the year 1893 was $\$ 1,760,489,273$, an increase of only $1 \%$, which might easily have been brought about through the destruction of weeds, would have meant a saving to the farmers of the nation of about $\$ 17,000,000$ during that year alone." (1) "The simple cost of weed removal along the railways of the State of Ohio is placed by Stair at over half a million dollars per annum." (13) "The weeds found in cornfields annually cost the farmer of Iowa many thousands of dollars." (12) "Minnesota produces annually about $200,000,000$ bushels of small grain. A dockage of one pound per bushel (due to weeds) means a loss of $200,000,000$ pounds. Had the land been free of weeds the same amount of plant food, moisture and labor would have produced over 3,000,000 bushels of wheat or the equivalent in other grains. This makes an annual loss due to weeds of about $\$ 2,500,000$ or an annual rental of about 30 cents an acre on every acre on which small grain is grown. Added to this great loss we must include cost of fighting weeds, loss of fertility and moisture, strain on machinery, extra cost of twine to tie up the weeds, freight charges for shipping weeds, etc." (14) In Ontario the "Bureau of Industries for the Province in 1898 sent out a few questions about weeds to its regular correspondents, and others, chiefly those who had done satisfactory experimental work in connection with the Experimental Union. "A large number of answers were received. . . A number estimate their loss at $25 \mathrm{c}$ per acre, and quite a few place it as high as $\$ 5$ per acre; so considering the whole list and counting labor, with the loss of soil moisture, fertility, etc., we think that $\$ 1$ per acre is a conservative estimate of the annual loss throughout the Province." (5)

C L. Marlatt in "The Annual Loss Occasioned by Destructive Insects in the United States" estimates the yearly tax chargeable to in- 
sects in this country as $\$ 795,100,000$. (10) "The common schools of the country cost in 1902 the sum of $\$ 235,000,000$, and all higher institutions of learning cost less than $\$ 50,000,000$, making the total cost of education in the United States considerably less than the farmers lost from insect ravages. . . . Furthermore, the yearly losses from insect ravages aggregate nearly twice as much as it costs to maintain our army and navy; more than twice the loss by fire; twice the capital invested in manufacturing agricultural implements; and nearly three times the estimated value of the products of all the fruit orchards, vineyards, and the small fruit farms in the country."

Prof. H. T. Fernald, Massachusetts State Entomologist, says: "Estimates of the annual loss by insects calculated at $18 \%$ are now considered as about correct, and this loss on the basis of the United States'government crop estimates for 1906 would be considerably over a billion dollars each year." (2)

If we were wise enough as a people to protect and increase our weed destroying insectivorous birds, they should largely control these enemies of our crops. Bobwhites, if we only had enough of them, ought to save us more than half of our $\$ 17,000,000$ weed damage, and of our billion dollar insect tax.

\section{Summary}

The bobwhite is known to eat 129 different kinds of weed seeds.

A single bird was found to eat as many as $12,000,18,000$ and 30,000 seeds of one kind of weed in a day.

They eat 15 grams, or half an ounce, of weed seed daily throughout the winter.

The known list of insects eaten - 135 - includes many of the most injurious species.

A single bird ate at one meal 568 mosquitoes; another during a day ate 1,350 flies; a third ate 5,000 plant lice, while still another record is 1,532 insects, 1,000 of which were grasshoppers.

Bobwhites eat from 12 to 24 grams of insects daily in the summer.

In a study of the growth and feeding of one bobwhite, it was found that in his third week he ate half of his weight of insects, in his fourth week one third. In the sixth the addition of grain brought it up to one half again. When adult they eat from one twelfth to one sixth of their weight.

An estimate of the average amount eaten by a bobwhite in a year is 2,732 grams, or about 5 pounds, of insects, and 4,681 grams, or abcut $93 / 4$ pounds, of weed seeds, which are equivalent to 65,302 insects and 5,123,076 weed seeds. 
I wish to acknowledge my indebtedness to Dr. C. F. Hodge of Clark University, under whose direction this work was done, for help and suggestions; to Mr. Arthur Merrill of the Massachusetts State Hatchery at Sutton, for assistance in rearing the birds; to Dr. F. M. Webster of the United States Bureau of Entomology, for supplying me with various insects; and to Mr. A. D. Selby of the Ohio Agricultural Experiment Station, and Mr. Geo. H. Clark, Seed Commissioner of the Department of Agriculture, Canada, for sending me weed seeds.

\section{Bibliography}

The numbers in the text refer to the numbered references below.

1. Coville, Frederick V. Letter of transmittal, Bul. No. 17. Legis̄ation against weeds. Div. of Botany, U. S. Dept. of Agriculture. 1896. p. 3. $60 \mathrm{p}$.

2. Fernald, H. T. Future of Economic Entomology. Popular Science Monthly. Feb. 1908.

3. Folsom, Justus W. Entomology with special reference to its Biological and Economic Aspects. Quoted from Slingerland. 1906. Philadelphia, p. Blakiston's Son \& Co. p. 394.485 p.

4. Harris, Edward. The Quail. The Best Insect and Weed Exterminator Must be Better Protected. Wm. Briggs, Publisher. Toronto, Ontario. 1905. p. $4.11 \mathrm{p}$.

5. Harrison, E. C. The Weeds of Ontario. Ontario Agricultural College. Dept. of Agriculture, Toronto. 1909. pp. 8-9. 80 p.

6. Judd, Sylvester D. The Bobwhite and other Quails of the United States in their Economic Relations. Bul. No. 21. Bureau of Biological Survey, U. S. Dept. of Agr. 1905. p. 31. 66 p.

7. The same as 6. p. 27.

8. Judd, Sylvester D. The Economic Value of the Bobwhite. Reprint from the Yearbook of the U. S. Dept. of Agr. 1903. pp. 195-196. $11 \mathrm{p}$.

9. The same as 6 . pp. $37-38$.

10. Marlatt, C. L. Annual Loss Occasioned by Destructive Insects in the United States, Burealu of Entomology, Reprint from Yearbook of Dept. of Agr. for 1904. p. 464.13 p.

11. Nash, Charles W. The Birds of Ontario in their relation to Agriculture. Bul. 173. Ontario Dept. of Agr. Toronto. 1909. 4th ed. p. 91. $95 \mathrm{p}$.

12. Pammel, L. H. Some Weeds of Iowa. Bul. 70. Exp. Sta. Iowa State College, Ames, Iowa. 1903. p. 300.233 p.

13. Selby, A. D. A Second Ohio Weed Manual. Bul. 175. Ohio Agr. Exp. Sta. Wooster, Ohio. 1906. p. 293. 384 p.

14. Wilson, A. D. Some Common Weeds and their Eradication. Bul. 95. Univ. of Minnesota Agr. Exp. Str., St. Anthony Park, Minn. 1907. p. 195. $43 \mathrm{p}$. 